\title{
ON THE ORIGIN OF THE HIERARCHY OF COLOR NAMES
}

\author{
VITTORIO LORETO \\ Physics Dept., Sapienza University of Rome, \\ Piazzale Aldo Moro 5, 00185 Roma, Italy and \\ Institute for Scientific Interchange (ISI), Viale Settimio Severo 65, 10133 Torino, Italy \\ vittorio.loreto@romal.infn.it \\ ANIMESH MUKHERJEE \\ Institute for Scientific Interchange (ISI), \\ Viale Settimio Severo 65, 10133 Torino, Italy \\ animesh.mukherjee@isi.it \\ FRANCESCA TRIA \\ Institute for Scientific Interchange (ISI), \\ Viale Settimio Severo 65, 10133 Torino, Italy \\ tria@isi.it
}

One of the fundamental problems in cognitive science is how humans categorize the visible color spectrum (Berlin \& Kay, 1969). The empirical evidence of the existence of universal or recurrent patterns in color naming across cultures is paralleled by the observation that color names begin to be used by individual cultures in a relatively fixed order (Berlin \& Kay, 1969). The origin of this hierarchy is largely unexplained. Here we resort to multi-agents simulations (Puglisi, Baronchelli, \& Loreto, 2008; Baronchelli, Gong, Puglisi, \& Loreto, 2010), where a population of individuals, subject to a simple perceptual constraint shared by all humans, namely the human Just Noticeable Difference, categorizes and names colors through a purely cultural negotiation in the form of language games (Steels $\&$ Belpaeme, 2005; Puglisi et al., 2008). We found that if one ranks different regions of the visible spectrum according to the time needed for a population to reach consensus on a color name for each specific region, color spectrum regions get organized into a hierarchy with [red, (magenta)-red], [violet], [green/yellow], [blue], [orange] and [cyan] appearing in this order (see Fig. 1). The hierarchy observed in our modeling scheme features an excellent quantitative agreement with the empirical observations of the World Color Survey (Berlin \& Kay, 1969; Cook, Kay, \& Regier, 2005; Baronchelli et al., 2010). Our results demonstrate a clear possible route to the emergence of hierarchical color categories, confirming that 
the theoretical modeling in this area has now attained the required maturity to make significant contributions to the ongoing debates concerning language universals (Roberson, Davies, \& Davidoff, 2000; Evans \& Levinson, 2009).

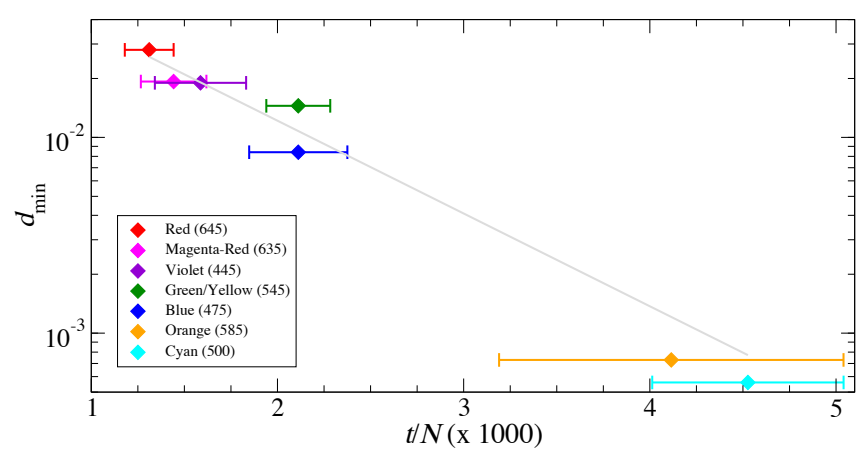

Figure 1. Time needed to reach consensus versus the value of Just Noticeable Difference, $d_{\min }$, for different regions of the color space corresponding to the colors reported in the legend (the approximate wavelength $(\mathrm{nm})$ associated with each colored data point is within parenthesis). Time is measured as number of games per agent ( $N$ is the population size). The gray lines in both the plots represent a fit of the respective data with an exponential function of the form $A e^{-\alpha t}$.

\section{References}

Baronchelli, A., Gong, T., Puglisi, A., \& Loreto, V. (2010). Modelling the emergence of universality in color naming patterns. Proc. Natl. Acad. Sci. USA (PNAS), 107, 2403-2407.

Berlin, B., \& Kay, P. (1969). Basic Color Terms. Berkeley: University of California Press.

Cook, R., Kay, P., \& Regier, T. (2005). The world color survey database: history and use. In H. Cohen \& C. Lefebvre (Eds.), Handbook of categorisation in the cognitive sciences. (pp. 224-242). Amsterdam and London: Elsevier.

Evans, N., \& Levinson, S. C. (2009). The myth of language universals: Language diversity and its importance for cognitive science. Behavioral and Brain Sciences, 32(05), 429-448.

Puglisi, A., Baronchelli, A., \& Loreto, V. (2008). Cultural route to the emergence of linguistic categories. Proc. Natl. Acad. Sci. USA (PNAS), 105(23), 7936.

Roberson, D., Davies, I., \& Davidoff, J. (2000). Color categories are not universal: Replications and new evidence from a stone-age culture. Journal of Experimental Psychology: General, 129(N/A), 369-398.

Steels, L., \& Belpaeme, T. (2005). Coordinating perceptually grounded categories through language: A case study for colour. Behavioral and Brain Sciences, 28(04), 469-489. 